\title{
SAINT-VENANT'S TORSION OF THIN-WALLED NONHOMOGENEOUS OPEN ELLIPTICAL CROSS SECTION
}

\author{
István Ecsedi \\ professor emeritus, University of Miskolc, Institute of Applied Mechanics \\ Miskolc-Egyetemváros, 3515 Miskolc, Hungary,e-mail: mechecs@uni-miskolc.hu \\ Ákos József Lengyel \\ assistant lecturer, University of Miskolc, Institute of Applied Mechanics \\ Miskolc-Egyetemváros, 3515 Miskolc, Hungary, e-mail: mechlen@uni-miskolc.hu \\ Attila Baksa \\ associate professor, University of Miskolc, Institute of Applied Mechanics \\ Miskolc-Egyetemváros, 3515 Miskolc, Hungary, e-mail: mechab@uni-miskolc.hu \\ Dávid Gönczi \\ assistant lecturer, University of Miskolc, Institute of Applied Mechanics \\ Miskolc-Egyetemváros, 3515 Miskolc, Hungary, e-mail: mechgoda@uni-miskolc.hu
}

\begin{abstract}
This paper deals with the Saint-Venant's torsion of thin-walled isotropic nonhomogeneous open elliptical cross section whose shear modulus depends on the one of the curvilinear coordinates which define the cross-sectional area of the beam. The approximate solution of torsion problem is obtained by variational method. The usual simplification assumptions are used to solve the uniform torsion problem of bars with thin-walled elliptical cross-sections. An example illustrates the application of the derived formulae of shearing stress and torsional rigidity.
\end{abstract}

Keywords: Saint-Venant's torsion, thin-walled, open cross section, nonhomogeneous

\section{Introduction}

The Saint-Venant's torsion of elastic thin-walled beam with open cross section is a usual topic of elasticity textbooks (Saada, 1974), (Timoshenko, et al., 1970), (Lurie, 1970), (Lebejzon, 1943), (Wang, 1953), (Vlasov, 1961), (Sun, 2006), (Oden, 1967). For thin-walled open cross section the solution of torsion problem is based on the membrane analogy and the approximate solution of uniform torsion of narrow rectangular cross section with sides $a$ and $t$ in which $a$ is much longer than $t$ (Saada, 1974), (Timoshenko et al., 1970), (Lurie, 1970), (Sun, 2006). Fig. 1 shows a homogeneous thin-walled rectangular cross section whose Prandtl stress function is denoted by $U=U(x, y)$. The approximate solution of uniform torsion of this homogeneous cross section is derived from the following boundary value problem (Saada, 1974), (Lurie, 1970), (Vlasov, 1961), (Sun, 2006), (Oden, 1967)

$$
\begin{gathered}
\frac{\partial^{2} U}{\partial y^{2}}=-2 G, \quad 0<t<1, \\
U(0)=U(t)=0 .
\end{gathered}
$$



In

It is important hypothesis that the Prandtl's stress function $U$ does not depend on the $x$ coordinate.

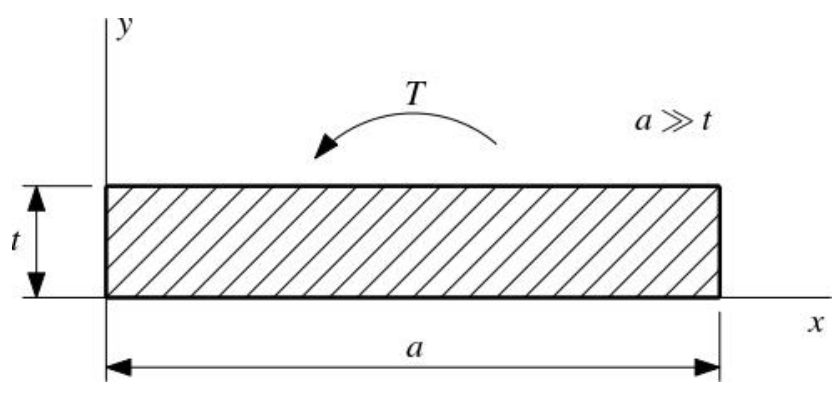

Figure 1. Narrow rectangular cross section

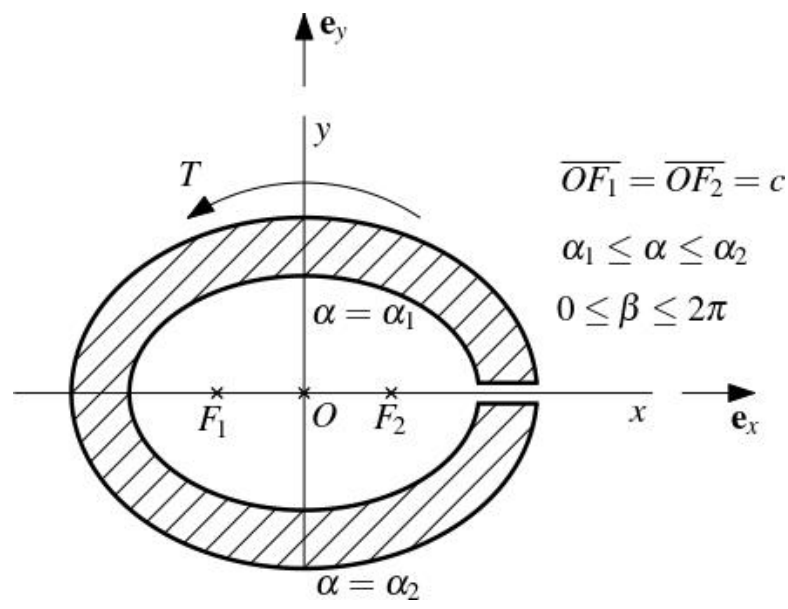

Figure 2. Thin-walled open elliptical cross section

Eq. (1) $G$ denotes the shear modulus of the material of homogeneous beam with narrow rectangular cross section. The approximate solution of the uniform torsion in this case is as follows (Saada, 1974), (Timoshenko et al., 1970), (Lurie, 1970), (Vlasov, 1961), (Sun, 2006)

$$
\begin{gathered}
U(y)=G\left(y t-y^{2}\right), \\
\tau_{y z}=0, \quad \tau_{x z}=\vartheta \frac{\partial U}{\partial y}=\vartheta G(t-2 y), \\
\vartheta=\frac{T}{S}, \quad S=G \frac{a t^{3}}{3} .
\end{gathered}
$$

In the above equation $\tau_{x z}$ and $\tau_{y z}$ are the shearing stresses, $\vartheta$ is the rate of twist with respect to the axial coordinate of the beam, $S$ is the torsional rigidity of the considered thin-walled rectangular cross section. In this paper an approximate solution of Saint-Venant's torsion is presented for thinwalled nonhomogeneous open elliptical cross section. Fig. 2 shows the considered open thin-walled 
elliptical cross section. To give the boundary contours of elliptical cross section we introduce an orthogonal curvilinear coordinate system $O \alpha \beta$ (Fig. 3)

$$
x=c \cosh \alpha \cos \beta, \quad z=c \sinh \alpha \sin \beta .
$$

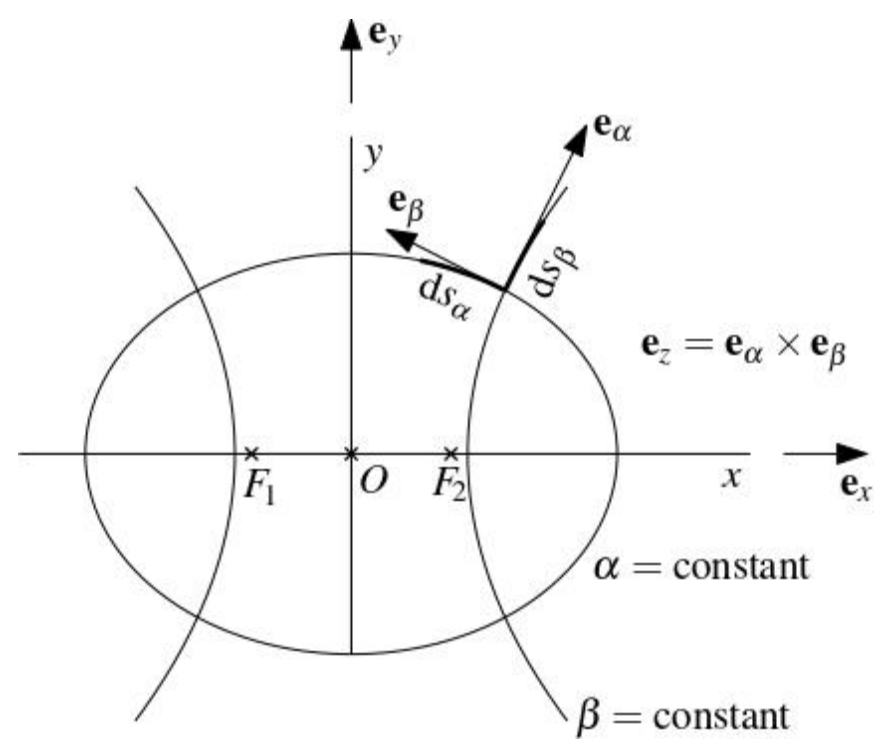

Figure 3. The coordinate lines of elliptical coordinate system $O \alpha \beta$

The focuses of boundary contour ellipse are the points $F_{1}$ and $F_{2}$ and theirs distance is $2 c$. In Cartesian coordinates $x$ and $y$ the equation of the curved boundary of the considered thin-walled cross section is

$$
\frac{x^{2}}{c^{2} \cosh ^{2} \alpha_{i}}+\frac{y^{2}}{c^{2} \sinh ^{2} \alpha_{i}}=1, \quad(i=1,2) .
$$

The coordinate lines of the elliptical coordinate system $O \alpha \beta$ are ellipses and hyperbolas as shown in Fig. 3

The unit vectors of $\alpha=$ constant and $\beta=$ constant coordinate lines are (Saada, 1974), (Slivker, 2007)

$$
\begin{gathered}
\mathbf{e}_{\beta}=\frac{1}{h}\left(-\cosh \alpha \sin \beta \mathbf{e}_{x}+\sinh \alpha \cos \beta \mathbf{e}_{y}\right), \\
\mathbf{e}_{\alpha}=\frac{1}{h}\left(\sinh \alpha \cos \beta \mathbf{e}_{x}+\cosh \alpha \sin \beta \mathbf{e}_{y}\right), \\
h^{2}=\cosh ^{2} \alpha-\cos ^{2} \beta .
\end{gathered}
$$

The expression of the area element $\mathrm{d} A$ is as follows

$$
\mathrm{d} A=\mathrm{d} x \mathrm{~d} y=c^{2} h^{2} \mathrm{~d} \alpha \mathrm{d} \beta .
$$


The two-dimensional del operator $\nabla$ can be represented as

$$
\nabla=\frac{\partial}{\partial x} \mathbf{e}_{x}+\frac{\partial}{\partial y} \mathbf{e}_{y}=\frac{1}{c h}\left(\frac{\partial}{\partial \alpha} \mathbf{e}_{\alpha}+\frac{\partial}{\partial \beta} \mathbf{e}_{\beta}\right) .
$$

\section{Governing equations and approximate solution}

Denote $U=U(\alpha, \beta)$ the Prandtl's stress function of the thin-walled open nonhomogeneous cross section. According to the variational formulation of Saint-Venant's torsion we know that $U=U(\alpha, \beta)$ can be obtained as the solution of the following variational problem (Slivker, 2007)

$$
\begin{gathered}
F(\tilde{U}(\alpha, \beta))=4 c^{2} \int_{\alpha_{1}}^{\alpha_{2}} \int_{0}^{2 \pi} \tilde{U}(\alpha, \beta)\left(\cos ^{2} \alpha-\sin ^{2} \beta\right) \mathrm{d} \alpha \mathrm{d} \beta- \\
-\int_{\alpha_{1}}^{\alpha_{2} 2 \pi} \frac{1}{G(\alpha, \beta)}\left[\left(\frac{\partial \tilde{U}}{\partial \alpha}\right)^{2}+\left(\frac{\partial \tilde{U}}{\partial \beta}\right)^{2}\right] \mathrm{d} \alpha \mathrm{d} \beta, \\
F(u(\alpha, \beta))=\min _{\tilde{U}(\alpha, \beta)} F(\tilde{U}(\alpha, \beta)),
\end{gathered}
$$

where the statically admissible Prandtl's stress function satisfies a homogeneous boundary condition on the whole boundary of nonhomogeneous thin-walled elliptical cross section, that is we have

$$
\begin{gathered}
\tilde{U}\left(\alpha_{1}, \beta\right)=\tilde{U}\left(\alpha_{2}, \beta\right)=0, \quad 0 \leq \beta \leq 2 \pi, \\
\tilde{U}(\alpha, 0)=\tilde{U}(\alpha, 2 \pi)=0, \quad \alpha_{1} \leq \alpha \leq \alpha_{2} .
\end{gathered}
$$

The approximation solution of the considered Saint-Venant's torsion is based on the following two assumptions

1. The Prandtl's stress function does not depend on the curvilinear coordinate $\beta$, that is

$$
U=U(\alpha)
$$

2. The boundary conditions $(16)_{1,2}$ are not satisfied

3. The shear modulus does not depend on $\beta$, that is

$$
G=G(\alpha) \text {. }
$$

Combination of equation (13) with equation (17) gives

$$
\hat{U}(\alpha, \beta)=U(\alpha), \quad \alpha_{1} \leq \alpha \leq \alpha_{2}, \quad 0 \leq \beta \leq 2 \pi
$$

and

$$
F[U(\alpha)]=4 c^{2} \int_{\alpha_{1}}^{\alpha_{2}} \int_{0}^{2 \pi} U(\alpha)\left(\cosh ^{2} \alpha-\cos ^{2} \beta\right) \mathrm{d} \alpha \mathrm{d} \beta-2 \pi \int_{\alpha_{1}}^{\alpha_{2}} \frac{1}{G(\alpha)}\left(\frac{\mathrm{d} U}{\mathrm{~d} \alpha}\right)^{2} \mathrm{~d} \alpha .
$$

According to the stationary condition of Timoshenko functional (13) under the condition 


$$
U\left(\alpha_{1}\right)=U\left(\alpha_{2}\right)=0, \quad 0 \leq \beta \leq 2 \pi
$$

it can be shown that $U=U(\alpha)$ is the solution of the undermentioned ordinary differential equation

$$
\frac{\mathrm{d}}{\mathrm{d} \alpha}\left(\frac{1}{G(\alpha)} \frac{\mathrm{d} U}{\mathrm{~d} \alpha}\right)+c^{2} \cosh 2 \alpha=0 .
$$

The solution of the boundary value problem given by equation (21) and (22) is

$$
U(\alpha)=\frac{c^{2}}{2}\left[\frac{\int_{\alpha_{1}}^{\alpha} G(\eta) \mathrm{d} \eta}{\int_{\alpha_{1}}^{\alpha_{2}} G(\eta) \mathrm{d} \eta^{\alpha_{2}}} \int_{\alpha_{1}}^{\alpha_{1}} G(\eta) \sinh 2 \eta \mathrm{d} \eta-\int_{\alpha_{1}}^{\alpha} G(\eta) \sinh 2 \eta \mathrm{d} \eta\right], \quad \alpha_{1} \leq \alpha \leq \alpha_{2} .
$$

The torsional rigidity of the thin-walled elliptical cross section is obtained from equation (24)

$$
S=2 \int_{A} U \mathrm{~d} A=2 c^{2} \int_{0}^{2 \pi} \int_{\alpha_{1}}^{\alpha_{2}} U(\alpha)\left(\cosh ^{2} \alpha-\cos ^{2} \beta\right) \mathrm{d} \alpha \mathrm{d} \beta .
$$

A detailed computation gives

$$
S=\frac{2 c^{4} \pi}{\int_{\alpha_{1}}^{\alpha_{2}} G(\alpha) \mathrm{d} \alpha^{\alpha_{1}}} \int_{\alpha_{2}}^{\alpha_{1}}\left(Q_{1}(\alpha)+Q_{2}(\alpha) \cosh ^{2} \alpha\right) \mathrm{d} \alpha
$$

where

$$
\begin{gathered}
Q_{1}(\alpha)=\frac{1}{2}\left[\int_{\alpha_{1}}^{\alpha_{2}} G(\eta) \mathrm{d} \eta \int_{\alpha_{1}}^{\alpha} G(\eta) \sinh 2 \eta \mathrm{d} \eta-\int_{\alpha_{1}}^{\alpha} G(\eta) \mathrm{d} \eta \int_{\alpha_{1}}^{\alpha_{2}} G(\eta) \sinh 2 \eta \mathrm{d} \eta\right], \\
Q_{2}(\alpha)=\cosh ^{2} \alpha\left[\int_{\alpha_{1}}^{\alpha} G(\eta) \mathrm{d} \eta \int_{\alpha_{1}}^{\alpha_{2}} G(\eta) \sinh 2 \eta \mathrm{d} \eta-\int_{\alpha_{1}}^{\alpha_{2}} G(\eta) \mathrm{d} \eta \int_{\alpha_{1}}^{\alpha} G(\eta) \sinh 2 \eta \mathrm{d} \eta\right] .
\end{gathered}
$$

For given applied torque the rate of twist $\vartheta$ is obtained as

$$
\vartheta=\frac{T}{S}
$$

The expression of shear stress vector in terms of $U=U(\alpha)$ is as follows (Fig. 1)

$$
\boldsymbol{\tau}_{z}=\vartheta \nabla U \times \mathbf{e}_{z}=\frac{\vartheta}{c \sqrt{\cosh ^{2} \alpha-\cos ^{2} \beta}} \frac{\partial U}{\partial \alpha} \mathbf{e}_{\alpha} \times \mathbf{e}_{z}=\frac{\vartheta}{c \sqrt{\cosh ^{2} \alpha-\cos ^{2} \beta}} \frac{\partial U}{\partial \alpha} \mathbf{e}_{\beta} .
$$

Combination of equation (28) with equation (29) gives for $\tau_{\beta}=\tau_{\beta}(\alpha, \beta)$ 


$$
\tau_{\beta}(\alpha, \beta)=\frac{c G(\alpha) T}{2 S \sqrt{\cosh ^{2} \alpha-\cos ^{2} \beta}}\left[\frac{\int_{\alpha_{1}}^{\alpha_{2}} G(\eta) \sinh 2 \eta \mathrm{d} \eta}{\int_{\alpha_{1}}^{\alpha_{2}} G(\eta) \mathrm{d} \eta}-\sinh 2 \alpha\right] .
$$

The zero-curve of the shearing stresses where

$$
\tau_{\beta}(\alpha, \beta)=0
$$

is an ellipse between the boundary ellipses on which $\alpha=\alpha_{1}$ and $\alpha=\alpha_{2}$. The equation of zero-curve is

$$
\frac{x^{2}}{c^{2} \cosh ^{2} \alpha_{0}}+\frac{y^{2}}{c^{2} \sinh ^{2} \alpha_{0}}=1
$$

here

$$
\alpha_{0}=\frac{1}{2} \operatorname{arsinh}\left[\frac{\int_{\alpha_{1}}^{\alpha_{2}} G(\eta) \sinh 2 \eta \mathrm{d} \eta}{\int_{\alpha_{1}}^{\alpha_{2}} G(\eta) \mathrm{d} \eta}\right]
$$

\section{Example}

In the numerical example the following data are used

$c=20 \mathrm{~mm}, \quad \alpha_{1}=0.46, \quad \alpha_{2}=0.5, G_{0}=2 \times 10^{4} \mathrm{MPa}, \lambda=0.5, \quad G(\alpha)=G_{0} \exp (\lambda \alpha), \quad T=1000 \mathrm{Nmm}$. Fig. 4 shows the plot of the Prandtl's stress function for $\alpha_{1} \leq \alpha \leq \alpha_{2}$

The graphs of the shearing stresses $\tau_{\beta}(\alpha, \beta)$ for $\alpha=\alpha_{1}, \quad \alpha=\alpha_{2}$ and $\alpha=\alpha_{0} \quad 0 \leq \beta \leq 2 \pi$ are shown in Fig. 5.

The value of the curvilinear coordinate of the zero stress curve is

$$
\alpha_{0}=0.4801659
$$

and the approximate value of the torsional rigidity is

$$
S=3.05735008 \times 10^{5} \mathrm{Nmm}^{2} .
$$

In this example the shear stress resultant at $\beta=0$ and $\beta=2 \pi$ is

$$
f_{\beta}=\int_{\alpha_{1}}^{\alpha_{2}} \tau_{\beta}(\alpha, 0) c \sqrt{\cosh ^{2} \alpha-1} \mathrm{~d} \alpha=2.888574 \times 10^{-7} \frac{\mathrm{N}}{\mathrm{mm}},
$$

which shows that the stress free boundary condition at the inclusion in weak form is satisfied. 


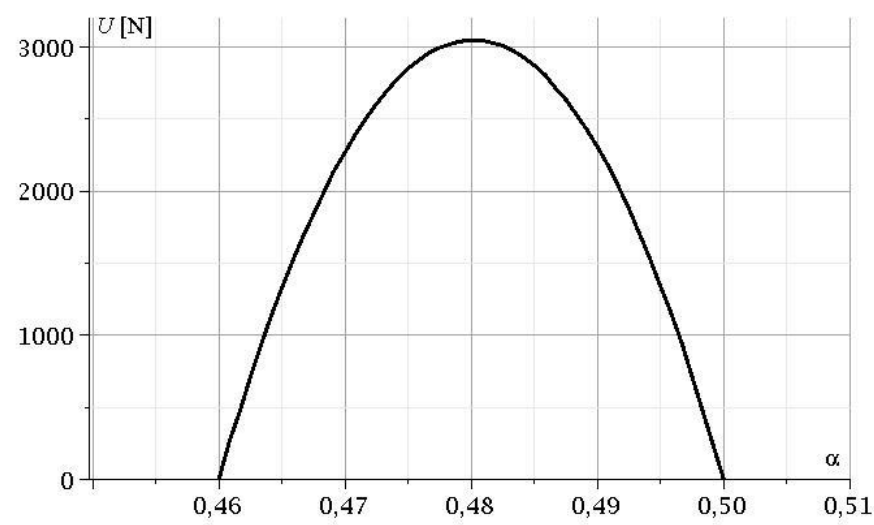

Figure 4. The graph of the Prandtl's stress function

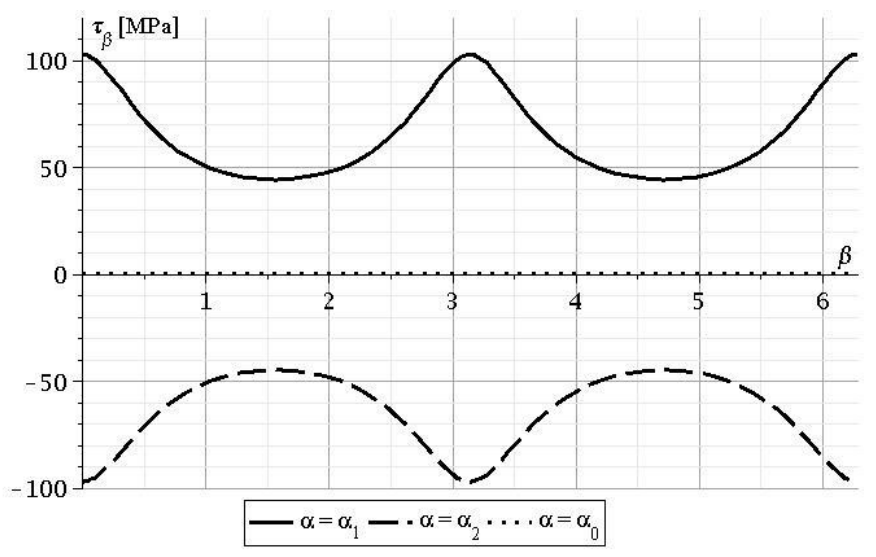

Figure 5. The graphs of the shearing stresses as a function of $\beta$

\section{Conclusions}

An approximate analytical solution is presented for the Saint-Venant's torsion of thin-walled isotropic nonhomogeneous open elliptical cross section. Timoshenko's variational method is used to derived the expression of the Prandtl's stress function. The usual assumptions for thin-walled open cross section bars gain adaptations to solve the considered uniform torsion problem.

\section{Acknowledgements}

This research was supported by the National Research, Development and Innovation Office - NKFIH, K115701.

This research was also carried out as part of the EFOP-3.6.1-16-2016-00011 Younger and Renewing University - Innovative Knowledge City - institutional development of the University of Miskolc aiming at intelligent specialisation" project implemented in the framework of the Szechenyi 2020 program. The realization of this project is supported by the European Union, co-financed by the European Social Fund. 


\section{References}

[1] Saada, A. S. (1974). Elasticity: Theory and Applications. Pergamon Press, New York.

[2] Timoshenko, S. P., Goodier, J. N. (1970). Theory of Elasticity. McGraw-Hill, New York.

[3] Lurie, A. I. (1970). Theory of Elasticity. Nauka, Moscow (in Russian).

[4] Lebejzon, L. S. (1943). Solution of Problems of Elasticity by Variational Methods. Gosztehizdat, Moscow (in Russian).

[5] Wang, C. T. (1953). Applied Elasticity. McGraw-Hill, New York.

[6] Vlasov, V. Z. (1961). Thin-Walled Elastic Beams. English translation: Oldbourne Press, London.

[7] Sun, C. T. (2006). Mechanics of Aircraft Structures. (Second edition) John Wiley and Sons Inc. New Jersey.

[8] Oden, J. T. (1967). Mechanics of Elastic Structures. McGraw-Hill.

[9] Renton, J. D. (1999). Elastic Beams and Frames. Camford Books, Oxford.

[10] Slivker, V. (2007). Mechanics of Structural Elements: Theory and Applications. SpringerVerlag, Berlin. https://doi.org/10.1007/978-3-540-44721-4 